\title{
Risk of active tuberculosis in immigrants: effects of age, region of origin and time since arrival in a low-exposure setting
}

\section{Emma S McBryde MB BS, PhD, FRACP Head of Epidemiology, and Principal Research Fellow \\ Justin T Denholm BMed, MPHTM, FRACP Infectious Diseases Physician,' and PhD Scholar \\ 1 Victorian Infectious Diseases Service, Melbourne Health, Melbourne, VIC \\ 2 Department of Medicine University of Melbourne Melbourne, VIC \\ Emma.McBryde@ mh.org.au}

doi: 10.5694/mjal2.10035 MJA 2012; 197: 458-461

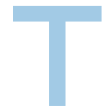
uberculosis (TB) is a major global problem, with 8.8 million cases and 1.1 million deaths in HIV-negative people estimated for 2010. ${ }^{1}$ Globally, the cornerstone of TB control is providing directly observed therapy to limit treatment failure and transmission. But in Australia and other low-incidence countries, most cases of TB are due to reactivation of latent infection and are preventable. Accordingly, supplementing directly observed therapy with diagnosis and treatment of latent TB infection is recommended for and used in high-risk groups for prevention of TB. ${ }^{2-5}$

Evaluation of public health strategies targeting latent TB infection has been limited by lack of accurate assessment of the risk of reactivation. To determine whether treatment of latent TB infection is worthwhile in a given setting, a critical starting point is accurate assessment of the risk of reactivation in those likely to have latent infection. Early studies of close contacts in high-prevalence settings suggest a $10 \%$ lifetime risk of reactivation, with half of cases occurring in the first 2 years following exposure. ${ }^{6}$ However, this estimate has recently been questioned in the era of low TB transmission rates and improved health in developed countries such as the United States and Australia. ${ }^{7}$ In addition, studies of the risk of reactivation following latent TB infection in low-prevalence settings have been hampered by the lack of a gold standard diagnostic test. ${ }^{8}$

Previous studies of TB incidence in Australia have taken a descriptive case-series approach ${ }^{9,10}$ or have estimated aggregate incidence of TB in immigrant groups. ${ }^{11}$ These studies have provided information on the risk of $\mathrm{TB}$ but have not used the cohort data necessary to provide accurate dynamic risk estimates for TB reactivation. Other studies have used cohort data on Australian immigrants but have analysed a restricted group,

\begin{abstract}
Objective: To estimate the risk of active tuberculosis (TB) in immigrants to Victoria, Australia, as a function of time since arrival and stratified by age group and region of origin.

Design, setting and subjects: Longitudinal study of the incidence of active TB in Victorian immigrants, excluding New Zealanders, who arrived in Australia between 1975 and 2007. Victorian immigration data were used to describe annual arrival cohorts by age and region of origin, and TB case notification data for 1995 to 2010 were matched to the cohorts. Survival analysis was performed to determine incidence rates of active TB.
\end{abstract}

Main outcome measures: Incidence of active TB, stratified by age, region of origin and time since arrival in Australia.

Results: Incidence of active TB following immigration to Australia was highly dependent on region of origin, with the highest risks being in immigrants from South Asia and sub-Saharan Africa. For immigrants from high-risk regions (Asia, Africa and the Pacific), the incidence of active TB infection was 100-150 per 100000 person-years in the first 6 years following immigration. Overall, we estimate that $50 \%$ of TB occurred within 7 years of arrival. A bimodal age distribution was evident, with peaks of incidence in 20-24-year-olds and 70-74-year-olds.

Conclusions: Our data show that substantial risk of TB in Victorian immigrants persists well beyond the first 2-3 years following arrival, and that risk is highly dependent on region of origin. The regional dependence suggests that public health strategies would benefit from more refined regional stratification of risk, which could be used to determine risk thresholds for the use of TB prevention strategies and predict rates of TB in Australia following particular patterns of immigration.

such as refugees ${ }^{12-14}$ or immigrants from a particular country of origin. ${ }^{15,16}$

We aimed to quantify the dynamic risk of active TB in immigrants based on age, region of origin and time since arrival in Australia. We describe a longitudinal cohort of immigrants to Victoria, to estimate changes in $\mathrm{TB}$ incidence as a function of time since arrival.

\section{Methods}

Data on immigration to Victoria were obtained from the Australian Bureau of Statistics (ABS) to define annual arrival cohorts from 1975 to 2007. Each annual cohort consisted of all immigrants to Victoria with data collected on age (in 5-year groupings), region of origin (as defined by the ABS) and year of arrival in Australia. Immigrants were included regardless of QuantiFERON assay, tuberculin skin test or chest radiography results.
The TB database of the Victorian Department of Health (formerly called the Department of Human Services, Victoria) was searched to extract all notified cases of TB from 1995 to 2010. Age at notification, region of origin and year of arrival in Australia were obtained for each notified case. Data on TB notifications were excluded if the notification occurred before 1995 (owing to incomplete records) or if the year of arrival was before 1975. Notifications relating to people immigrating from New Zealand were not included in the study as denominator data are not reflected in the ABS immigration figures owing to the large number of New Zealanders in Australia not seeking immigration status.

All analysed data were de-identified before extraction, and used with approval from the relevant data managers. According to Melbourne Health policy, ethics review was not required for the study. 


\section{Statistical methods}

The data were analysed using a survival analysis with proportional hazards assumptions. To develop a survival analysis with the data, the aggregate immigration data were transformed into individual-level data. To estimate unobserved attrition in the cohort, death of a proportion of the cohort was incorporated by assuming that, after arrival in Australia, immigrants adopted the Australian age-matched population risk of death. ${ }^{17}$

Once the entire cohort of immigrants was specified, those for whom a case of TB had been notified were added. A matched group of immigrants (matched according to age group at arrival, year of arrival and region of origin) was deleted from the initial cohort to avoid double entry of those who went on to develop TB.

Survival was defined as TB-free survival. The entry point for individuals included in the analysis was 1995 or year of arrival, whichever was the later. For those arriving before 1995, the origin point of the risk was taken as the arrival year. For example, a person who immigrated in 1990 would have been included in the analysis from 1995 but assumed to be at risk of TB for 5 years before entry into the analysis.

Classical survival analysis was used to determine the incidence rate of active TB infection. The exit point from the study was year of notification for those who developed active TB, or the end of 2010 for those who did not. A Cox proportional hazard assumption was used to estimate the effect of region on risk of active TB infection and this was tested using Schoenfeld residuals. ${ }^{18}$ The associated factors tested in the proportional hazards analysis were age and region of origin. Age was defined as age at entry into the analysis and grouped into 5-year categories. At-risk years (denominators for measure of risk) were adjusted for expected deaths in the cohort of immigrants. This adjustment was according to age at study entry, following Australian life expectancy tables. ${ }^{17}$ A sensitivity analysis was used to examine the estimated TB incidence rate in each age group under different attrition assumptions. Calculations were performed using
STATA/SE 11.0 (StataCorp LP). Cumulative risk of TB over the study period was graphed in Matlab 2010b (MathWorks). Further details regarding sensitivity analysis methods and outcomes are provided in the Appendix (online at mja.com.au).

\section{Results}

The total number of immigrants who arrived in Victoria from 1975 to 2007, excluding New Zealanders, was 673434. We estimate that this provided 8.9 million at-risk years over the study period (1995-2010). During the study period, 5347 notifications of active TB were made to the Victorian Department of Health, of which 3712 had recorded information that pertained to the cohort of immigrants with documented arrival times between 1975 and 2007, 3689 of whom had all relevant data fields completed.

The median age of immigrants at arrival was 26 years (interquartile range, 13-35 years), and the median age at arrival for those who subsequently developed active TB was 27 years (interquartile range, 21-38 years). Most immigrants came from Asia $(47 \%, 315812)$ or Europe (31\%, $210170)$. Of those 3689 immigrants who later developed active $\mathrm{TB}$, most were from Asia $(75 \%, 2777)$ and Africa $(18 \%, 649)$, and $2 \%$ (72) were from the Pacific.

The incidence rate of active TB in Victorian immigrants from high-risk areas - Asia, Africa and the Pacific was estimated to be 100-150 per 100000 person-years for the first 6 years following arrival (Box 1). This rate fell to about 50 per 100000 person-years at 12 years following arrival, and then settled at about 25 per 100000 person-years. Within the high-risk regions there are areas of higher and lower incidence. For example, immigrants from South Asia and sub-Saharan Africa have the highest incidence estimated in this study: 270 and 190 per 100000 person-years, respectively, in the first 2 years after arrival. Cumulative risk of active TB (Box 2) shows the marked regional differences. The regions with the lowest risk were Europe and the Americas.

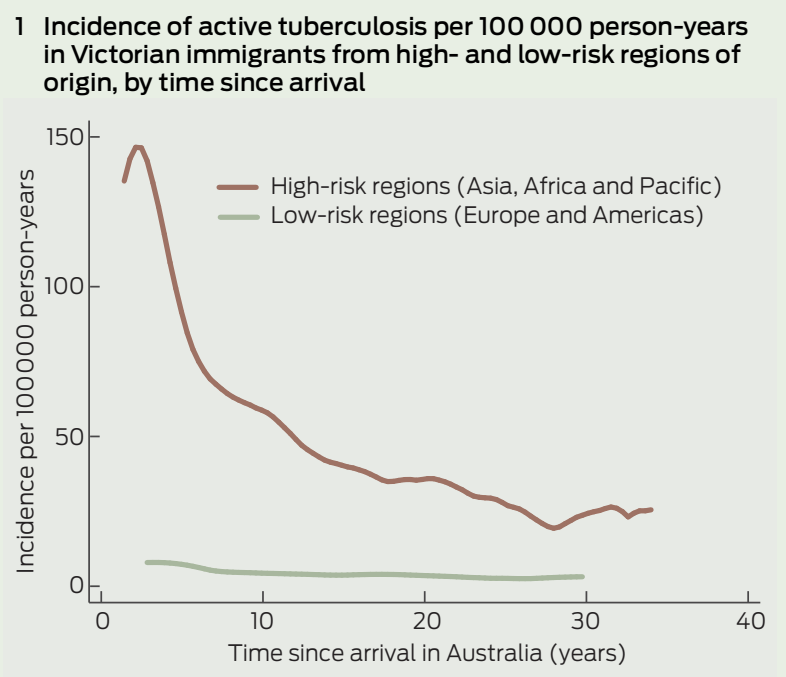

In addition, the risk varies as a function of time since arrival - the highest incidence is in the first 2 years, but elevated incidence persists beyond that period. Of those who develop active TB during the first 35 years following arrival, we estimated that $50 \%$ present within 7 years and only $20 \%$ present within 2 years.

When TB incidence was stratified by age, a bimodal pattern was seen incidence peaked in 20-24-year-olds and 70-74-year-olds (Box 3). A complete table of hazard ratios for $\mathrm{TB}$ estimated in a multivariable model by region of origin and age group are given in the Appendix.

\section{Discussion}

Our study shows that the risk of active TB is moderately high in Australian immigrants, and that this rate is age dependent and reflects incidence in regions of origin.

The incidence of active TB in the 6 years following arrival is about 100150 per 100000 person-years for high-risk immigrant groups. The incidence decreases in the subsequent decade, but it remains higher - for at least 35 years after immigration than the estimated incidence for the Australian born population (1.5 per 100000 person-years $^{19}$ ). The risk of active TB is age dependent, with a bimodal peak in incidence in the 20 s and the 70s. This finding differs from previous observations, which have not shown a higher rate of $\mathrm{TB}$ in older people. ${ }^{20,21}$ Half the risk of TB occurred within 7 years of arrival to 


\section{Cumulative risk of active tuberculosis in Victorian immigrants, by time since arrival and region of origin}

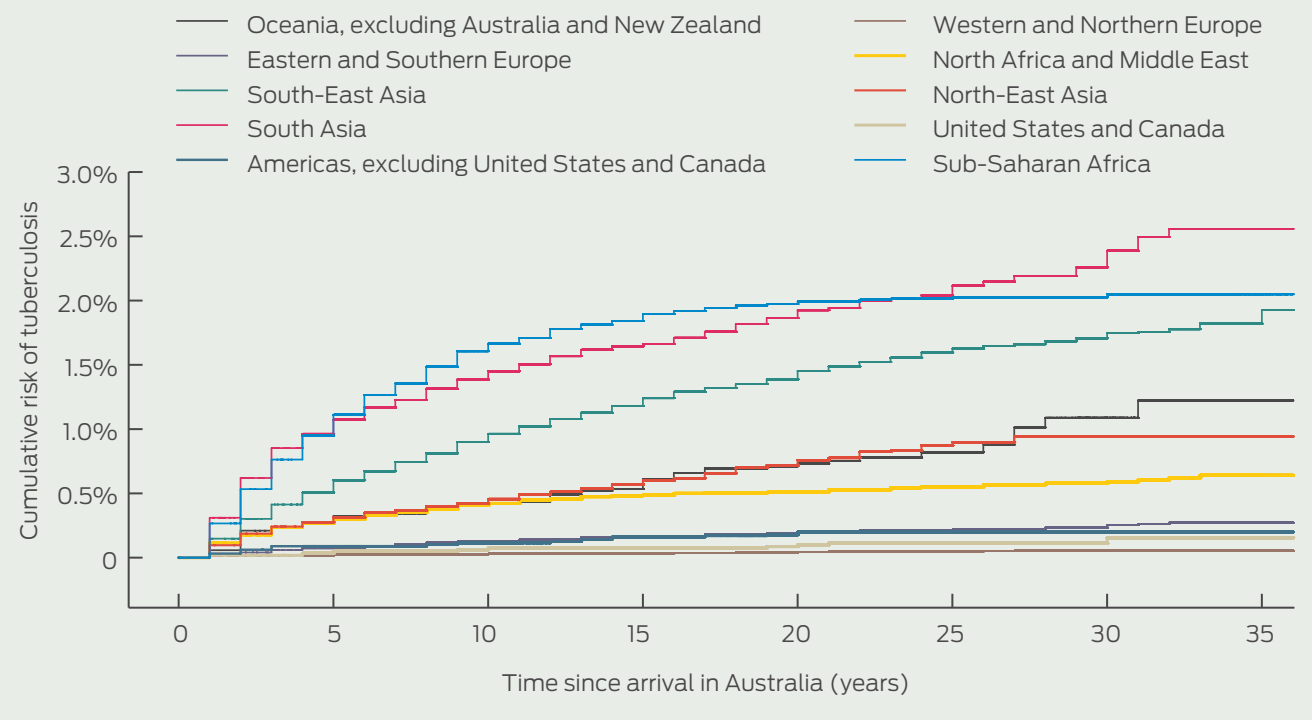

Victoria and 20\% within the first 2 years - a similar result to that of an earlier study, in which half the risk was in the first 5 years and $30 \%$ was in the first 2 years. ${ }^{19}$

A strength of our study was the establishment of an immigration cohort as the denominator for assessing the absolute risk per person-year of active TB infection. This enabled assessment of dynamic incidence rates of $\mathrm{TB}$ as a function of age, region of origin and time since arrival. A limitation of our study was its retrospective nature; however, continuous statewide data collections were used to minimise reporting bias. Intrastate travel following migration to Australia could introduce discrepancy between locations of immigration and TB notifications.

3 Incidence of active tuberculosis per 100000 person-years in Victorian immigrants, by age group

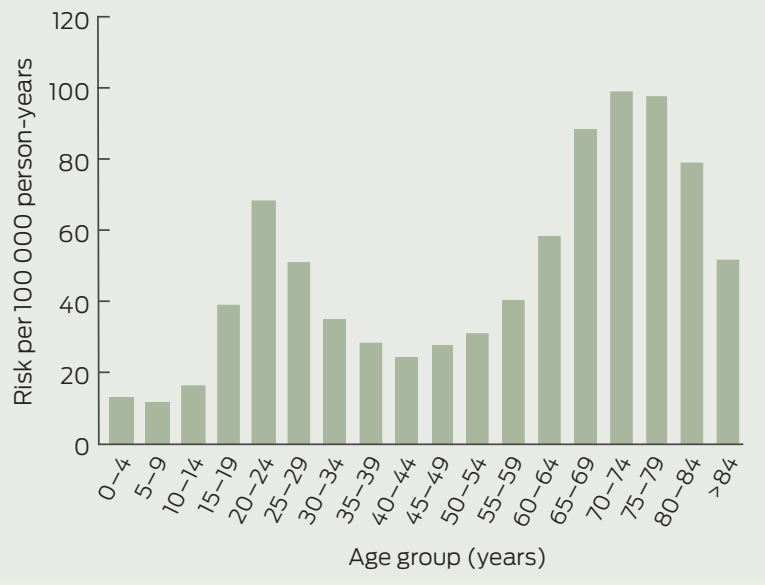

Sensitivity analysis shows that if the net rates of migration out of Victoria are much higher than anticipated, there would be a substantially higher estimate of risk of TB in older people and those in Australia for greater than 10 years (see Appendix). In addition, the sensitivity analysis provides a robust lower estimate of incidence for older age groups, predicting a peak in incidence in 70-74-year-olds that is similar in magnitude to the peak seen in 20-24-year-olds. Hence, if this immigrant cohort has death rates lower than the Australian average (as observed in other Australian immigrant cohorts $^{20}$ ), the risk of TB in the older age-groups will be only marginally overestimated in our study.

Regardless of the assumptions put into the sensitivity analysis, the qualitative conclusions are the same: (a) the risk of TB is age dependent, with a second peak in older people, and (b) the incidence rate of $\mathrm{TB}$ is highest after arrival but remains above the Australian rate for some decades. There are some residual limitations intrinsic to the data sources used in this analysis. In particular, denominator data were derived from immigration statistics and do not capture short-term visitors from overseas. Additional information on movement of immigrants interstate and on shortterm visitors would have improved the accuracy of our estimates.

Region of origin is an important predictor of TB risk. Rates of TB incidence in our study cohort on arrival are similar to the reported incidence rates in the countries of origin. For example, immigrants from South Asia and subSaharan Africa have the highest incidence estimated in this study - 270 and 190 per 100000 person-years respectively in the first 2 years after arrival. This compares with World Health Organization estimates of incidence within these regions of 165-277 (India-Pakistan) and 276 (sub-Saharan Africa) per 100000 person-years. ${ }^{22}$ Our estimates of incidence in South-East Asia are higher than those estimated using Victorian notification data from 1987 to $1992 .{ }^{19}$ This earlier study estimated an incidence of 47.5 per 100000 person-years for this region, compared with our estimate of 65 per 100000 person-years (1430 cases over 2200000 person-years) in South-East Asia. This discrepancy could reflect a different mix of immigrants to the region over time, with changes in immigration patterns and higher rates of refugee intake from South-East Asia in recent years.

Age is also an important predictor of risk. Risk of active TB in our study peaked in the 20s, as in other studies. ${ }^{21}$ Incidence in under 5-year-olds was very low in our study compared with incidence of TB in native populations, ${ }^{21}$ this probably reflects a very low risk of transmission in the Victorian immigrants. Incidence in older people was very high in our study, a feature that has been absent in many studies of developing countries. ${ }^{21,23}$ While the data are sparse for older age groups, leading to uncertainty regarding the true incidence in older people, the effect size is large, with a relative risk greater than the peak observed in the 20-24-year-olds.

These findings have several consequences for physicians and public health policymakers in Australia. First, we must revise the notion that risk of active $\mathrm{TB}$ is principally in the first 2-3 years following arrival in Australia. Second, the marked regional variation in incidence suggests that countries and regions of origin need more refined stratification than simply high, medium and low incidence of TB. Finally, the efficacy and cost-effectiveness of treatment of latent TB infection is highly dependent on risk of reactivation, so the 
findings in our study could be used to determine risk thresholds for the use of various public health strategies for preventing $\mathrm{TB}$, as contact tracing and genotyping indicate that the vast majority of cases notified in Australia are due to reactivation rather than autochthonous transmission. ${ }^{24}$

Our results could also be incorporated into a dynamic model to predict rates of TB in Australia following particular patterns of immigration (ie, specific regions of origin and numbers of immigrants). Such modelling could be used to inform policies regarding strategies to reduce TB in Australia, while avoiding unnecessary testing in low-risk populations. Although low by global standards, the TB incidence in Victoria is 70 times higher than the WHO Millennium Development Goal target. ${ }^{22}$ This situation is unlikely to improve without implementation of well targeted public health strategies that include treatment of latent TB infection.

Acknowledgements: Justin Denholm is an National Health and Medical Research Council Postgraduate Scholarship recipient for doctoral studies in TB. We thank the Victorian Department of Health for providing TB notification data; in particular, we thank Lynne Brown for collecting and managing the notification data.

Competing interests: No relevant disclosures.

Received 9 Jan 2012, accepted 22 Jul 2012.

1 WHO global tuberculosis control report 2010. Summary. Cent Eur J Public Health 2010; 18: 237.

2 Denholm JT, McBrydeES. The use of antituberculosis therapy for latent TB infection. Infect Drug Resist 2010; 3: 63-72.
3 Denholm J, McBryde E. Management of latent tuberculosis infections in Australia and New Zealand: a review of current practice. Tuberc Res Treat 2010; 2010: 284028. doi: 10.1155/2010/ 284028

4 Murray RJ, Davis JS, Burgner DP, et al. The Australasian Society for Infectious Diseases guidelines for the diagnosis, management and prevention of infections in recently arrived refugees: an abridged outline. Med J Aust 2009; 190: 421-425

5 National Institute for Health and Clinical Excellence. Tuberculosis: clinical diagnosis and management of tuberculosis, and measures for its prevention and control. NICE clinical guidelines CG117. March 2011. http:// publications.nice.org.uk/tuberculosis-cgl17/ guidance (accessed Aug 2012).

6 Comstock GW, Ferebee SH, Hammes LM. A controlled trial of community-wide isoniazid prophylaxis in Alaska.Am Rev Respir Dis 1967; 95 : 935-943.

7 Horsburgh CR Jr, O’Donnell M, Chamblee S, et al. Revisiting rates of reactivation tuberculosis: a population-based approach. Am J Respir Crit Care Med 2010; 182: 420-425.

8 Pai M, Riley LW, Colford JM Jr. Interferon-gamma assays in the immunodiagnosis of tuberculosis: a systematic review. Lancet Infect Dis 2004; 4 : 761-776.

9 McPherson ME, Kelly H, Patel MS, Leslie D. Persistent risk of tuberculosis in migrants a decade after arrival in Australia. Med J Aust 2008; 188 : 528-531.

10 McPherson ME, Leslie D, Sievers A, et al. Epidemiology of laboratory confirmed tuberculosis in Victoria, 1990 to 2004. Commun Dis Intell 2008; 32: 237-241.

11 Watkins RE, Plant AJ. Predicting tuberculosis among migrant groups. Epidemiol Infect 2002; 129: 623-628.

12 Marks GB, Bai J, Simpson SE, et al. Incidence of tuberculosis among a cohort of tuberculinpositive refugees in Australia: reappraising the estimates of risk. Am J Respir Crit Care Med 2000; 162: 1851-1854.

13 Marks GB, Bai J, Stewart GJ, et al. Effectiveness of postmigration screening in controlling tuberculosis among refugees: a historical cohort study, 1984-1998. Am J Public Health 2001; 91: 1797-1799.

14 Marks GB, Britton WJ. Priorities for the treatment of latent tuberculosis. N Engl J Med 2004; 35l: 832-834.

15 Maclntyre CR, Plant AJ. Tuberculosis in SouthEast Asian refugees after resettlement - can prevention be improved by better policy and practice? Prev Med 1998; 27: 815-820.

16 Maclntyre CR, Plant AJ. Longitudinal incidence of tuberculosis in South-East Asian refugees afte re-settlement. Int J Tuberc Lung Dis 1999; 3 : 287-293.

17 Australian Bureau of Statistics. Life tables, Australia, 2003 to 2005. Nov 2006. (ABS Cat No. 3302.0.55.001.) http://www.abs.gov.au/ AUSSTATS/abs@.nsf/Lookup/ 3302.0.55.001Main+Featuresl2003\%20to\%202 005?OpenDocument (accessed Aug 2012).

18 Schoenfeld D. Partial residuals for the proportional hazards regression model. Biometrika 1982; 69: 239-241. doi: 10.1093/ biomet/69.1.239.

19 Maclntyre CR, Dwyer B, Streeton JA. The epidemiology of tuberculosis in Victoria. Med $J$ Aust 1993; 159: 672-677.

20 Singh M, de Looper M. Australian health inequalities: birthplace. Canberra: Australian Institute of Health and Welfare, 2002. (AlHW Cat. No. AUS 27.) http://www.aihw.gov.au/ publication-detail/?id=6442467357 (accessed Aug 2012).

21 Donald PR. Childhood tuberculosis: the hidden epidemic. Int J Tuberc Lung Dis 2004; 8: 627-629.

22 World Health Organization. Global tuberculosis control: WHO report 2011. Geneva: WHO, 2011. http://www.who.int/tb/publications/global report/2011/gtbrll_full.pdf (accessed Aug 2012).

23 Donald PR, Marais BJ, Barry CE 3rd. Age and the epidemiology and pathogenesis of tuberculosis. Lancet 2010; 375: 1852-1854.

24 Denholm JT, Leslie DE, Jenkin GA, et al. Longterm follow-up of contacts exposed to multidrug-resistant tuberculosis in Victoria, Australia, 1995-2010. Int J Tuberc Lung Dis 2012: Aug 3 [Epub ahead of print].

\section{Stamps of greatness}

\section{Julius Wagner von Jauregg (1857-1940)}

VON JAUREGG was born in Wels, Austria, in March 1857, and studied medicine at the University of Vienna, graduating in 1880. He became particularly interested in mental diseases, becoming a great neurologist and psychiatrist.

In 1893 he became director of the psychiatric clinic of the Provincial Hospital in Vienna. There he introduced the use of iodised salt for the prevention and treatment of endemic goitre and cretinism.

For 50 years he concentrated on the problem of neurosyphilis. In 1890, following the discovery of tuberculin by Koch, he began to use it to produce fever in the insane in the hope that artificially induced fever might have the same effect as he had seen in a patient with erysipelas.

Later, in 1914, he introduced the therapeutic use of malaria and found some success. In 1917, he began to inoculate paretic

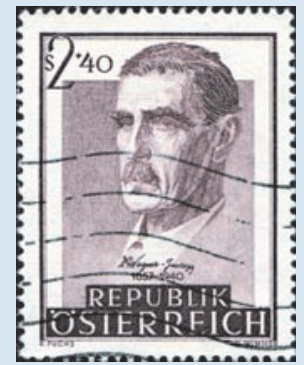

soldiers with malaria; cerebral syphilis had hitherto been incurable. He found that of his 86 patients, 21 were still alive, and seven working, after several years.

After 20 years he attained some degree of control of the hyperpyrexia created by the malarial therapy.

In 1927 he was awarded the Nobel Prize in Medicine.

It is of historical interest to note that the malarial treatment of syphilis had been used at the Bloomingdale Hospital in New York some 30 years before von Jauregg introduced it in Vienna.

He died in Vienna on 4 October 1940 and in 1957 on the 100th anniversary of his birth he was philatelically honoured by Austria. 